\title{
Radiation-induced modifications on physico chemical properties of diluted nitric acid solutions within advanced Spent Nuclear Fuel reprocessing
}

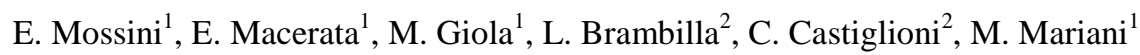 \\ ${ }^{1}$ Energy Department, Nuclear Engineering section - CeSNEF, Politecnico di Milano, Italy \\ ${ }^{2}$ Chemistry, Materials and Chemical Engineering "Giulio Natta", Politecnico di Milano, Italy
}

Within advanced spent nuclear fuel reprocessing, the effects of ionizing radiation on some physico chemical properties of $0.25-0.5 \mathrm{M} \mathrm{HNO}_{3}$ solutions have been studied for the first time, because of the possible influence on both the separation performances and the fluid-dynamics of the extracting system. Irradiations were performed in air up to $100 \mathrm{kGy}$ by two ${ }^{60} \mathrm{Co}$-sources, with $0.3 \mathrm{kGy} / \mathrm{h}$ and $2.5 \mathrm{kGy} / \mathrm{h}$ dose rates, respectively. Density, viscosity, acidity and nitrate ion concentration were measured before and after irradiation. No modifications of these properties in the dose range considered were observed, therefore no significant effects on the fluid-dynamics of the extracting systems are expected.

KEYWORDS: partitioning, $\gamma$-radiolysis, nitric acid, density, viscosity, acidity.

\section{INTRODUCTION}

In the last decades, the increasing radioactive waste amount coming from nuclear energy power plants has become such a big deal so as to encourage the development of innovative treatments with the aim to reduce the radiotoxicity and the heat production of the final waste which has to be stored in dedicated repository [1]. One of the possible approaches to be pursued is hydro-metallurgical partitioning of minor actinides coupled with transmutation in proper burner reactors in order to obtain shorter-lived or even stable nuclides [2,3], reducing the required storage time from 100000 years to few hundreds of years [4]. The most promising partitioning strategies are based on a three consecutive steps approach: i) separation of uranium and plutonium from spent nuclear fuel; ii) co-extraction of trivalent actinides and lanthanides; iii) separation of trivalent actinides from trivalent lanthanides [2]. The last step is of capital importance because several lanthanides are distinguished by large neutron capture cross sections [5] and their presence would impact on transmutation efficiency [6]. Since the last step is also the most difficult to be pursued due to the similar chemical behaviour of $4 \mathrm{f}$ and $5 \mathrm{f}$ elements, several hydrometallurgical processes have been proposed [7,8]. Among them, iSANEX (innovative Selective ActiNides EXtraction) process is one of the most promising. GANEX (Grouped ActiNides EXtraction) process is a valid alternative approach, since it would allow the separation of minor actinides in several oxidation states, not only the trivalent [7].

Since these systems will be employed in highly radioactive environments, mainly due to alpha-emitting radionuclides, the radiation chemistry of both ligands and diluents will play an essential role in determining extraction efficiency, separation factors and solvent-recycling. Radiation-induced effects would likely include ligand concentration reduction and production of degradation products of ligand and/or diluent [1]. It is known from the literature that most of the radiation damage falls on the diluent and, indirectly, on the ligands [9]. Therefore, it is paramount to primarily study the effects of radiolysis on the diluents [10], in order to foresee the possible interactions among the extractant and the diluent by-products as well as to evaluate changes of both the separation performances and the fluid-dynamics of the extracting system. These by-products would undesirably affect not only the efficiency of the separation process, but also the physico chemical properties of the solvent, hence leading to the formation of precipitates and third phases, thus inducing alterations of density and viscosity $[11,12]$. These modifications of the process streams could seriously impact the safety of the future reprocessing plants, due to malfunctioning at the pumps and at the centrifuges, so it acquires prominence a deep investigation of the effects of radiation on the physico chemical properties of the system. The effects of radiation on concentrated nitric acid solutions have been widely studied in the past since early ' 60 s because of its employment in the first separative processes (e.g. PUREX) [1]. A great effort has been done to evaluate the formation yields of stable by-products under alpha [13] and gamma irradiation conditions [10]. p-Nitroaniline was successfully introduced in the solutions in order to prevent the decomposition of the nitrite anion by-product and its reaction with $\mathrm{H}_{2} \mathrm{O}_{2}$ [14]. In the same work performed by Kazanjian et.al., the LET effect has been investigated in detail. Moreover, in order to better understand the by-products formation mechanism, a pulse radiolysis study has been performed [15]. Radiation chemistry of actinides in nitric acid solutions has implied great efforts and several works have been performed [16], also requiring the help of computational codes to model radiolysis of actinide ions [17]. More recently, several studies have been performed, changing the viewpoint in favour of the process safety, focusing the attention on the identification of harmful by-products $[18,19]$ and on the evaluation of density and viscosity alterations [20] of 30\% TBP-n-dodecane- $\mathrm{HNO}_{3}$. Concerning the streams involved in advanced spent nuclear fuel reprocessing, a lack of 
knowledge remains, in particular regarding the radiolytic behaviour of systems involving diluted nitric acid solution and organic mixture of kerosene/1-octanol.

The purpose of the present experimental work is to bridge this gap studying in depth the effects of radiation on physico chemical properties of diluted nitric acid solution in order to assess whether any modification of important physico chemical properties occurs, resulting in possible alterations of the fluid-dynamics of the extracting system. Such information would be of capital interest for the safety and the feasibility of the new and promising processes based on hydrophilic extractants, e.g. i-SANEX. For this aim density, viscosity, acidity and nitrate anion concentration $\left[\mathrm{NO}_{3}{ }^{-}\right]$of diluted nitric acid solutions were systematically measured before and after $\gamma$-irradiation, at dose rates and total absorbed doses of interest for the future industrial implementation.

\section{EXPERIMENTAL}

\subsection{REAGENTS AND MATERIALS}

All the analysed solutions were prepared with concentrated nitric acid (65\%, Fluka) and distilled water purified with Millipore ultrapurification system. All reagents used in the analyses are analytical grade and were used without further purifications.

\subsection{EXPERIMENTAL CONDITIONS}

$0.25 \mathrm{M}$ and $0.5 \mathrm{M} \mathrm{HNO}_{3}$ solutions were prepared from concentrated $\mathrm{HNO}_{3}$ and stored in the dark in glass vials sealed with plastic lid. Irradiation campaigns were performed in air using two ${ }^{60} \mathrm{Co}$-sources with approximately $0.3 \mathrm{kGy} / \mathrm{h}$ and $2.5 \mathrm{kGy} / \mathrm{h}$ dose rates respectively, up to total absorbed dose of 50kGy for the lower dose rate irradiation and 100kGy for the higher dose rate irradiation. These irradiation conditions are coherent with the conditions that a SANEX stream should undergo [21]. Following irradiation no volume decrease was measured, hence solvent evaporation is expected to be negligible. Then the samples were kept sealed at $4^{\circ} \mathrm{C}$ in the dark until further analyses.

\section{RESULTS AND DISCUSSION}

\subsection{DENSITY}

Density measurements on approximately $2.7 \mathrm{~mL}$ of fresh and irradiated solutions were performed by DMA $35 \mathrm{~N}$ Anton Paar portable density meter, which is affected by a measuring uncertainty of $\pm 0.001 \mathrm{~g} / \mathrm{cm}^{3}$, by keeping the samples in a thermostatic bath with temperature resolution of $\pm 0.1^{\circ} \mathrm{C}$. The samples were analysed at temperatures of interest for the separative process, ranging from $20^{\circ} \mathrm{C}$ to $45^{\circ} \mathrm{C}$, with incremental temperature of $5^{\circ} \mathrm{C}$. Each density value is the mean of 10 measurements. The estimation of the measuring uncertainty has been carried out according to Minimum Mean Square Error method, considering the variances of the acquired data and the uncertainty associated to the instrument itself [22].

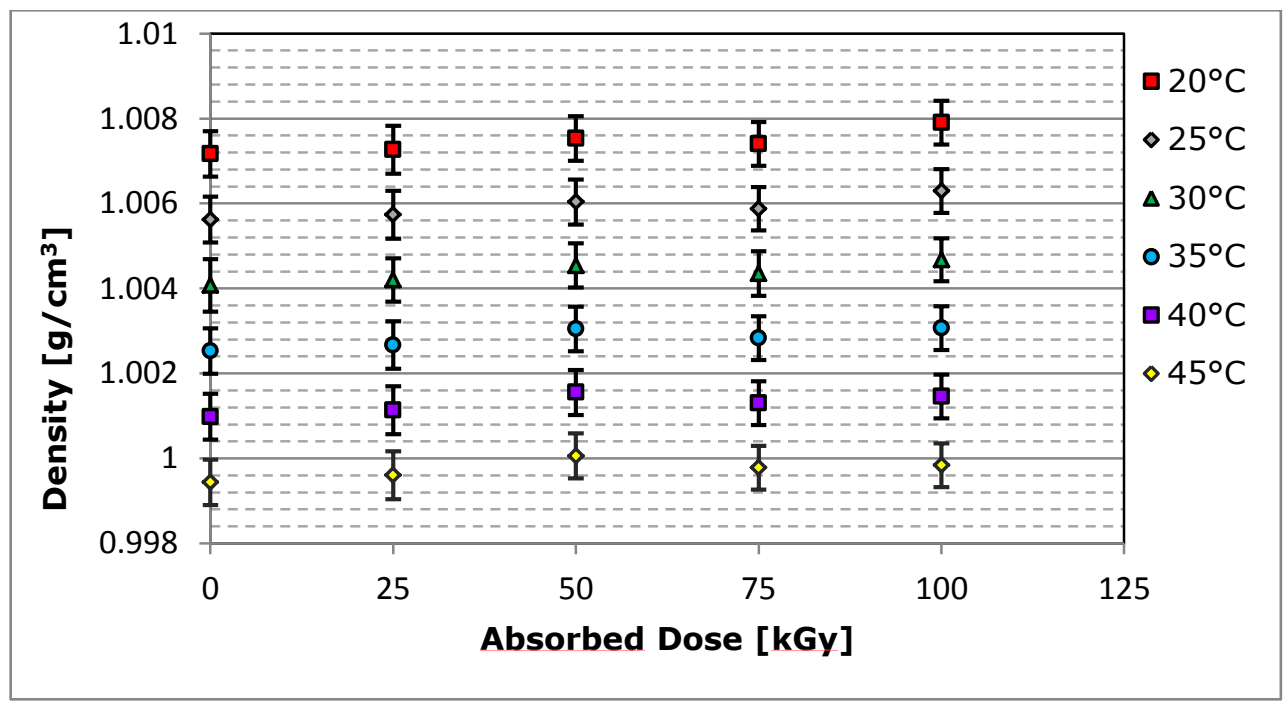

Fig 1 Density of $0.25 \mathrm{M} \mathrm{HNO}_{3}$ as a function of absorbed dose at high dose rate 


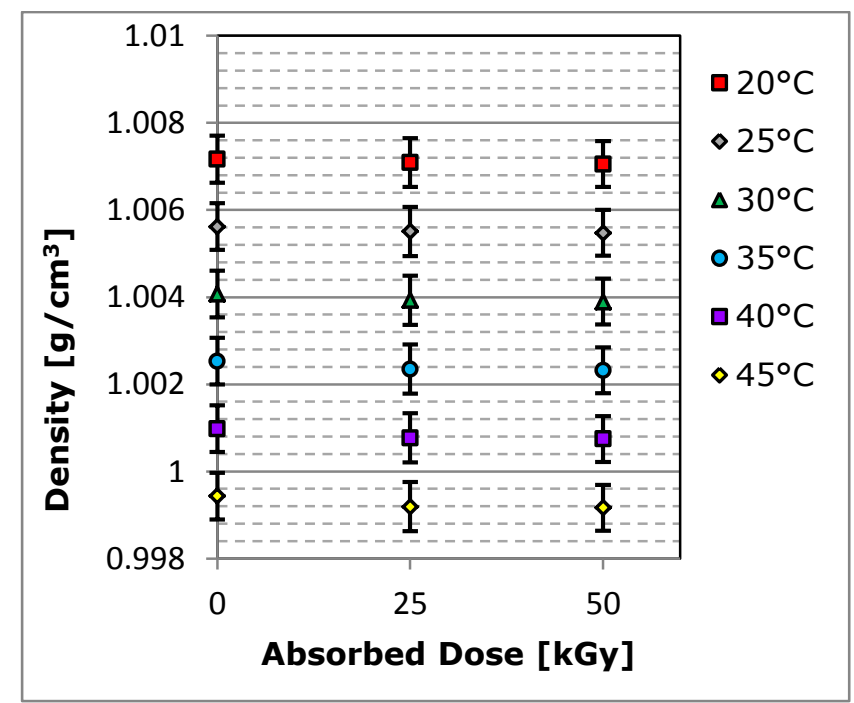

Fig 2 Density of $0.25 \mathrm{M} \mathrm{HNO}_{3}$ as a function of absorbed dose at low dose rate

The results obtained for $0.25 \mathrm{M} \mathrm{HNO}_{3}$ irradiated samples are reported in Fig 1 and in Fig 2. It could be observed that no density radiation-induced variation is noticeable for the absorbed dose range considered at both dose rates within the limit of experimental error. Similar trends were found for irradiated $0.5 \mathrm{M} \mathrm{HNO}_{3}$ solutions.

\subsection{VISCOSITY}

Viscosity measurements were performed using a KPG-UBBELOHDE micro-viscometer (0.32mm diameter capillary) on approximately $2.7 \mathrm{~mL}$ of fresh and irradiated solutions keeping the samples in a thermostatic bath with temperature resolution of $\pm 0.1^{\circ} \mathrm{C}$. The samples were analysed at temperature ranging from $20^{\circ} \mathrm{C}$ to $45^{\circ} \mathrm{C}$, with incremental temperature of $5^{\circ} \mathrm{C}$. In order to perform accurate measurements, an equilibration time of about 10 minutes was applied at each temperature step to enable the sample to reach a constant temperature. A digital stopwatch with time resolution of 1/100 second was used to measure the time interval the leading edge of the meniscus of the sample takes to descent from the upper timing mark to the lower one. The Hagenback correction was subtracted from the measured efflux time to obtain the corrected efflux time. Finally, the kinematic viscosity was obtained by multiplying this value for a constant coefficient K, typical of the micro-viscometer used.

The kinematic viscosity is the mean of 5 measurements, with an accuracy of $\pm 0.7 \%$, including the Hagenback correction and the errors related to temperature variations and micro-viscometer set-up. The estimation of the total measuring uncertainty has been carried out according to Minimum Mean Square Error method [22].

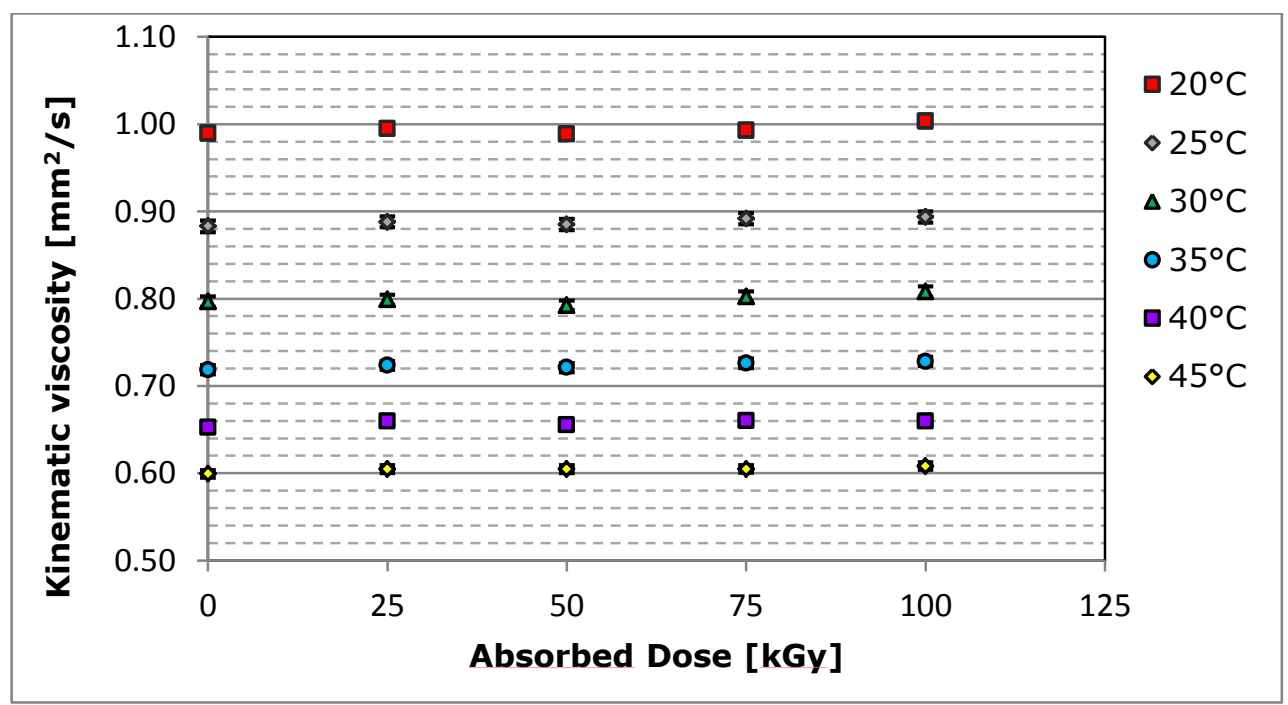

Fig 3 Kinematic viscosity of $0.25 \mathrm{M} \mathrm{HNO}_{3}$ as a function of absorbed dose at high dose rate 
The results obtained for irradiated $0.25 \mathrm{M} \mathrm{HNO}_{3}$ samples are reported in Fig 3 and in Fig 4. No kinematic viscosity radiation-induced variation is noticeable for the absorbed dose range considered within the limit of experimental error at both dose rates. Similar trends were found for irradiated $0.5 \mathrm{M} \mathrm{HNO}_{3}$ solutions.

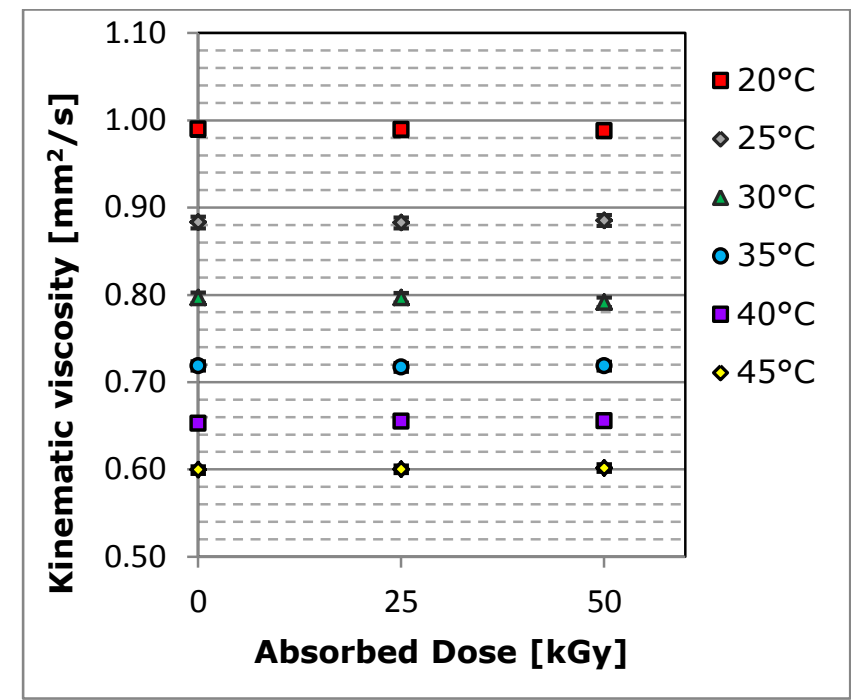

Fig 4 Kinematic viscosity of $0.25 \mathrm{M} \mathrm{HNO}_{3}$ as a function of absorbed dose at low dose rate

\subsection{ACIDITY}

Total acidity of approximately $5 \mathrm{~mL}$ of $0.5 \mathrm{M} \mathrm{HNO}$ and $10 \mathrm{~mL}$ of $0.25 \mathrm{M} \mathrm{HNO}_{3}$ was measured before and after irradiation by titration with $0.1 \mathrm{M} \mathrm{NaOH}$. Methyl orange was used as indicator to determine the end point of the titration. Acidity was measured with an uncertainty of $\pm 0.45 \%$.

Table $1 \mathrm{H}^{+}$concentration of $0.25 \mathrm{M}$ and $0.5 \mathrm{M} \mathrm{HNO}$ as a function of absorbed dose at high dose rate

\begin{tabular}{lccccc}
\hline & 0 kGy & 25 kGy & 50 kGy & 75 kGy & 100 kGy \\
\hline $\mathbf{0 . 2 5} \mathbf{M}$ & $0.250 \pm 0.001$ & $0.250 \pm 0.001$ & $0.250 \pm 0.001$ & $0.250 \pm 0.001$ & $0.251 \pm 0.001$ \\
$\mathbf{0 . 5} \mathbf{M}$ & $0.506 \pm 0.002$ & $0.507 \pm 0.002$ & $0.505 \pm 0.002$ & $0.504 \pm 0.002$ & $0.505 \pm 0.002$ \\
\hline
\end{tabular}

Table $2 \mathrm{H}^{+}$concentration $[\mathrm{M}]$ of $0.25 \mathrm{M}$ and $0.5 \mathrm{M} \mathrm{HNO}$ as a function of absorbed dose at low dose rate

\begin{tabular}{lccc}
\hline & 0 kGy & 25 kGy & 50 kGy \\
\hline $\mathbf{0 . 2 5} \mathbf{M}$ & $0.250 \pm 0.001$ & $0.251 \pm 0.001$ & $0.251 \pm 0.001$ \\
$\mathbf{0 . 5} \mathbf{M}$ & $0.499 \pm 0.002$ & $0.498 \pm 0.003$ & $0.499 \pm 0.003$ \\
\hline
\end{tabular}

The results obtained for $0.25 \mathrm{M}$ and $0.5 \mathrm{M} \mathrm{HNO}_{3}$ samples at both high and low dose rate are reported in Table 1 and in Table 2. No radiation-induced modifications are noticeable within the considered irradiation conditions.

\subsection{NITRATE ANION CONCENTRATION}

It is known from the literature that in diluted nitric acid solution the reaction between nitrate ions and water radiolytic products leads to nitrite ion formation [14,15]. Moreover, higher nitrate ion concentration solutions undergo increasing nitrite ion production [14]. Within the development of advanced separative processes, the evaluation of nitrite ion concentration is of fundamental importance, because this by-product directly affects the red ox chemistry of several actinides [23]. The widespread technique employed to determine the nitrite anion concentration is Gas Chromatography-Mass Spectrometry because of its high sensitivity [14]. Unfortunately, this by-product is difficult to 
measure in acidic solutions because it immediately decomposes or it reacts with $\mathrm{H}_{2} \mathrm{O}_{2}$ [14]. In order to overcome the disadvantages related to the nitrite anion evaluation, in this work two different techniques have been employed to measure the nitrate ion concentration.

\subsubsection{UV spectrophotometry}

UV/VIS spectrophotometric analyses of fresh and irradiated $\mathrm{HNO}_{3}$ solutions were performed by Lambda EZ210 UV/VIS spectrophotometer (PerkinElmer) in the wavelength range of $230 \div 600 \mathrm{~nm}$. All solutions were diluted with the same ratio (1:2) in order to read an optimal absorbance response and to introduce the same error due to dilution. Nitrate anion peak was recognised at $301 \mathrm{~nm}$. A calibration of nitrate anion concentration was obtained by measuring the $\mathrm{NO}_{3}{ }^{-}$ absorbance in solutions of $\mathrm{HNO}_{3}$ ranging from $0.15 \mathrm{M}$ to $0.6 \mathrm{M}$, with increasing concentration of $0.05 \mathrm{M}$.

The nitrate anion concentrations measured for $0.25 \mathrm{M}$ and $0.5 \mathrm{M} \mathrm{HNO}_{3}$ samples are reported in Table 3 and inTable 4 . In the case of $0.25 \mathrm{M} \mathrm{HNO}_{3}$ no modifications were observed, while, in the case of $0.5 \mathrm{M} \mathrm{HNO}_{3}$ a nitrate anion consumption of about $4.5 \%$ at $100 \mathrm{kGy}$ with high dose rate and of about $4 \%$ at $50 \mathrm{kGy}$ with low dose rate were observed. This result could be justified by the fact that higher nitrate ion concentration solutions experience higher nitrate anion consumption [14] and the UV/VIS spectrophotometric technique is not sensitive enough to highlight the $\mathrm{NO}_{3}{ }^{-}$decrease in the case of irradiated $0.25 \mathrm{M} \mathrm{HNO}_{3}$.

Table 3 Nitrate anion concentration in $0.25 \mathrm{M}$ and $0.5 \mathrm{M} \mathrm{HNO}$ as a function of absorbed dose at high dose rate

\begin{tabular}{lccccc}
\hline & 0 kGy & 25 kGy & 50 kGy & 75 kGy & 100 kGy \\
\hline $\mathbf{0 . 2 5} \mathbf{M}$ & $0.242 \pm 0.001$ & $0.243 \pm 0.001$ & $0.244 \pm 0.001$ & $0.244 \pm 0.001$ & $0.241 \pm 0.001$ \\
$\mathbf{0 . 5} \mathbf{M}$ & $0.526 \pm 0.005$ & $0.516 \pm 0.005$ & $0.513 \pm 0.005$ & $0.502 \pm 0.005$ & $0.503 \pm 0.005$ \\
\hline
\end{tabular}

Table 4 Nitrate anion concentration in $0.25 \mathrm{M}$ and $0.5 \mathrm{M} \mathrm{HNO}$ as a function of absorbed dose at low dose rate

\begin{tabular}{lccc}
\hline & $\mathbf{0 ~ k G y}$ & $\mathbf{2 5} \mathbf{~ k G y}$ & $\mathbf{5 0} \mathbf{~ k G y}$ \\
\hline $\mathbf{0 . 2 5} \mathbf{M}$ & $0.250 \pm 0.001$ & $0.250 \pm 0.001$ & $0.249 \pm 0.001$ \\
$\mathbf{0 . 5} \mathbf{M}$ & $0.523 \pm 0.005$ & $0.505 \pm 0.005$ & $0.502 \pm 0.005$ \\
\hline
\end{tabular}

\subsubsection{RAMAN spectroscopy}

In order to further prove the results of UV/VIS spectrophotometric analyses, Raman spectra of approximately $0.2 \mathrm{~mL}$ of fresh and irradiated $\mathrm{HNO}_{3}$ in corked glass NMR tubes were acquired at room temperature. Each spectrum was collected as averaged result of four acquisitions 20 seconds each, in the range of $100 \div 3800 \mathrm{~cm}^{-1}$. Spectra analyses were carried out using Omnic 7.1 software.

The experimental apparatus consists of $\mathrm{Ar}^{+}$Laser Stabilite2017 from Spectra-Physics, 514.5nm excitation wavelength, $75 \mathrm{mWatt}$ power on sample; Labram HR800 Raman spectrometer from Horiba Jobin Yvon, coupled with Olympus BX41 microscope, 20X objective, 1024 pixel CCD thermo-electrically cooled, notch filter and 1800 groove/mm grating.

In all the spectra analysed water $\mathrm{OH}$ stretching, water $\mathrm{HOH}$ bending, nitrate and nitrite anion peaks are easily recognised [24]. In order to perform a semi-quantitative evaluation of the nitrate ion concentration, water bending peak was assumed as internal reference in both fresh and irradiated samples and the ratio between the nitrate ion peak and the water bending peak net areas were calculated. As shown in Fig 5 and in Fig 6, the ratios obtained for $0.25 \mathrm{M}$ and $0.5 \mathrm{M}$ $\mathrm{HNO}_{3}$ samples remain constant within the considered irradiation conditions. Therefore, no radiation-induced modification seem to occur. The results obtained with Raman spectroscopy seem to be in contradiction with the evidences of the UV/Vis spectrophotometric measurements concerning the $0.5 \mathrm{M} \mathrm{HNO}_{3}$ series. In order to achieve a deeper understanding, further investigations are already in progress, even resorting to ATR FT-IR spectroscopy and Ion Chromatography [25]. 


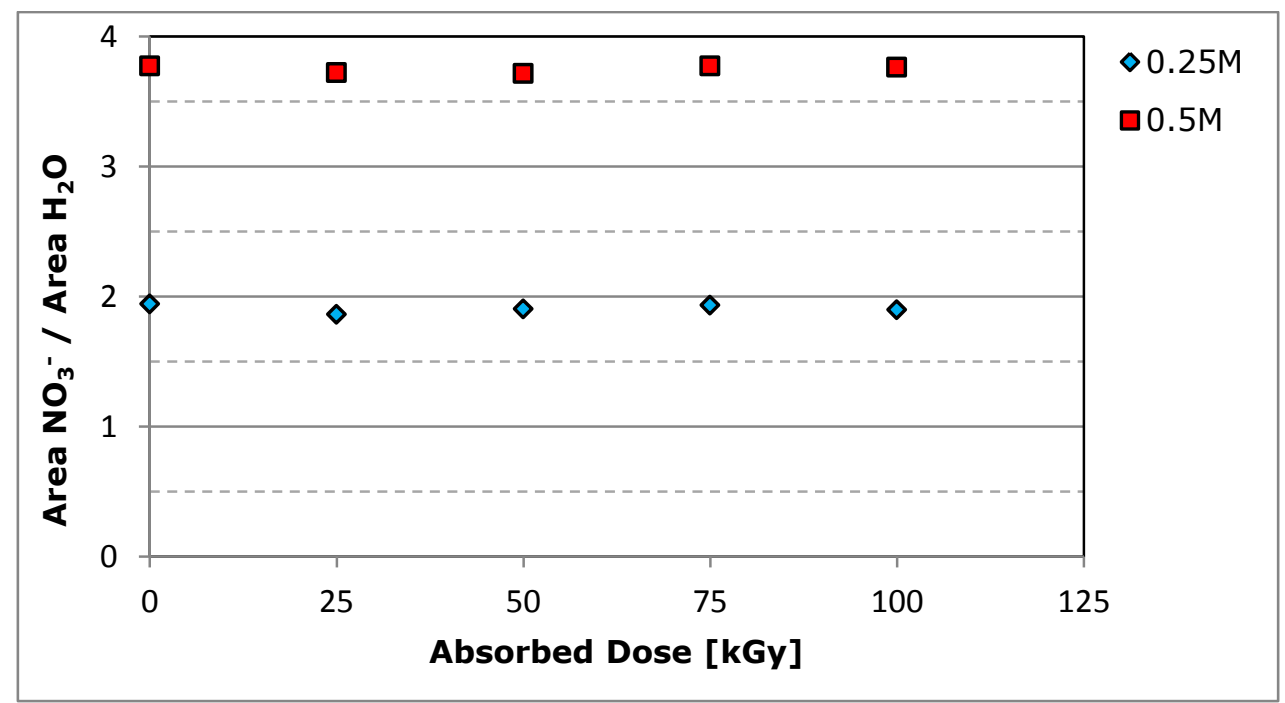

Fig 5 Ratio between nitrate anion and water bending peaks net areas of $0.25 \mathrm{M}$ and $0.5 \mathrm{M} \mathrm{HNO}$ as a function of absorbed dose at high dose rate

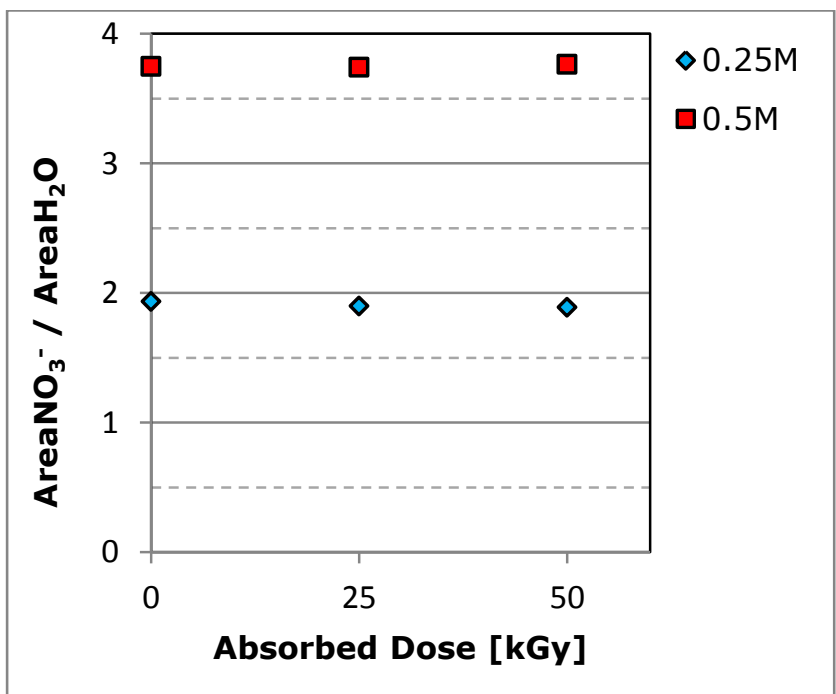

Fig 6 Ratio between nitrate anion and water bending peaks net areas of $0.25 \mathrm{M}$ and $0.5 \mathrm{M} \mathrm{HNO}$ as a function of absorbed dose at low dose rate

\section{CONCLUSION}

The radiolysis of concentrated nitric acid solutions and of organic diluent (e.g. TBP and n-dodecane) has been widely studied in the last decades within the PUREX process development $[1,18,19,20]$. Otherwise, the recent application of hydrophilic complexing ligands for the selective separation of actinides from the spent nuclear fuel [6,7] has led to the employment of diluted nitric acid solutions. Poor literature is present concerning the radiolytic behaviour of this innovative extracting system to be used in advanced spent nuclear fuel reprocessing processes (e.g. i-SANEX and GANEX) [11,26].

With the aim of evaluating the radiation induced effects on the fluid-dynamics of this innovative extracting system, in this experimental work a systematic analysis of physico chemical properties of diluted nitric acid solutions (such as density, viscosity and acidity) has been performed for the first time. The preliminary results of this study show that no variation of macroscopic physico chemical properties (such as density, viscosity and acidity) was observed in irradiated nitric acid solutions with increasing the absorbed dose in the range considered with respect to the un-irradiated reference sample. Therefore, no significant changes in the fluid-dynamics of the extracting system should be expected. This result is very encouraging for a future industrial-scale development. Concerning the nitrate anion concentration, a few percent consumption in the most concentrated solution is observed only by UV spectrophotometric technique and further investigations involving other spectroscopic and chromatographic techniques are already in progress. 
The study of radiation-induced modification of physico chemical properties will be extended from the aqueous diluent to the complete aqueous solvent, including the extracting ligand and, finally, to the whole system in which the hydrophilic ligand containing aqueous phase will be irradiated in contact with the organic phase. The analyses on the irradiated complete aqueous solvent proposed for the selective stripping of actinides are already in progress.

\section{AKNOWLEDGMENTS}

The authors wish to thanks Gammatom Srl and professor Armando Buttafava of Chemical Department of Università degli Studi di Pavia for the irradiation campaign.

This work was funded by the SACSESS European Commission Project (FP7-CP-2013-323282).

\section{REFERENCES}

[1] Mincher BJ, Modolo G, Mezyk SP (2009) The effects of radiation chemistry on solvent extraction 1: Conditions in acidic solutions and a review of TBP radiolysis. Solvent Extr Ion Exch 27:1-25

[2] Bourg S, Hill C, Caravaca C, Rhodes C, Ekberg C, Taylor R, Geist A, Modolo G, Cassayre L, Malmbeck R, Harrison M, de Angelis G, Espartero A, Bouvet S, Ouvrier N (2009) ACSEPT-Partitioning technologies and actinide science: Towards pilot facilities in Europe. Nucl Eng Des 241:3427-3425

[3] Salvatores M, Palmiotti G (2011) Radioactive waste partitioning and transmutation within advanced fuel cycles: Achievements and challenges. Prog Part Nucl Phys 66:144-166

[4] Magill J, Berthou V, Haas D, Galy J, Schenkel R, Wiese HW, Heusener G, Tommasi J, Youinou G (2003) Impact limits of partitioning and transmutation scenarios on nuclear waste isolation times. Nucl Ener 42:263-277

[5] Kopecky J (1997) Atlas of Neutron Capture Cross Sections. Report INDC (NDS)-362, IAEA, Vienna

[6] Panak P, Geist A (2013) Complexation and extraction of trivalent Actinides and Lanthanides by Triazinylpyridine N-Donor ligands. Chem Rev 113:1199-1236

[7] Bourg S, Poinssot C, Geist A, Cassayre L, Rhodes C, Ekberg C (2012) Advanced reprocessing developments in Europe: status on European projects ACSEPT and ACTINET-I3. Proc Chem 7:166-171

[8] Malmbeck R (2011) Advanced fuel cycle options. Energ Proc 7:93-102

[9] Pikaev AK, Gogolev AV, Shilov VP (1997) Radiation chemistry of aqueous solutions of actinides. Russ Chem Rev 66:763-788

[10] Katsumura Y, Jiang PY, Nagaishi R, Yotsuyanagi T, Ishigure K (1994) $\gamma$-Radiolysis study of concentrated nitric acid solutions. J Chem Soc Faraday Trans 90:93-95

[11] Mincher BJ Modolo G, Mezyk SP (2010) The effects of radiation chemistry on solvent extraction 4: Separation of the trivalent Actinides and considerations for radiation-resistant solvent system. Solvent Extr Ion Exch 28:415-436

[12] Pikaev AK, Kabakchi SA, Egorov GF (1988) Some radiation chemical aspects of nuclear engineering. Radiat Phys Chem 31:789-803

[13] Savel'ev YI, Ershova ZV, Vladimirova MV (1967) $\alpha$-Radiolysis of aqueous solutions of nitric acid. Soviet Radiochem., 9:221-225

[14] Kazanjian AR, Miner FJ, Brown AK, Hagan PG, Berry JW (1970) Radiolysis of nitric acid solutions: L.E.T. Effects. Trans Faraday Soc 66:2192-2198

[15] Katsumura Y, Jiang PY, Nagaishi R, Oishi T, Ishigure K, Yoshida Y (1991) Pulse radiolysis study of aqueous nitric acid solutions. Formation mechanism, yield, and reactivity of $\mathrm{NO}_{3}$ radical. J Phys Chem, 95:4435-4439

[16] Bhattacharyya PK, Natarajan, PR (1991) Radiation chemistry of actinide solutions. In: Freeman, A.J., Keller, C. (Eds.), Handbook on the Physics Chemistry of the Actinides. Elsevier Science Publishers B.V, Amsterdam, (Chapter 13).

[17] Nagaishi R (2001) A model for radiolysis of nitric acid and its application to the radiation chemistry of uranium ion in nitric acid medium. Radiat Phys Chem, 60:369-375

[18] Tripathi SC, Bindu P, Ramanujam A (2001) Studies on the identification of harmful radiolytic products of $30 \%$ TBP-n-dodecane- $\mathrm{HNO}_{3}$ by gas liquid chromatography. I. Formation of diluent degradation products and their role in Pu retention behavior. Separ Sci Technol 36:1463-1478 
[19] Krishnamurthy MV, Sampathkumar R (1992) Radiation-induced decomposition of the tributyl phosphate-nitric acid system: Role of nitric acid, J. Radioanal. Nucl. Chem. 166:421-429

[20] Tripathi SC, Ramanujam A (2003) Effect of radiation induced physicochemical transformation on density and viscosity of 30\% TBP - n-dodecane- $\mathrm{HNO}_{3}$ systems. Separ Sci Technol 38:2307-2326

[21] Magnusson D, Christiansen B, Malmbeck R, Glatz JP (2009) Investigation of the radiolytic stability of a CyMe $4^{-}$ BTBP based SANEX process, Radiochim Acta 97:497-502

[22] Taylor JR (1996) An Introduction to Error Analysis: The Study of Uncertainties in Physical Measurements. Univ Science Books, Sausalito

[23] Nash KL, Lumetta GJ (2011) Advanced separation techniques for nuclear fuel reprocessing and radioactive waste treatment. Woodhead Publishing Limited, Cambridge

[24] Aksenenko VM, Murav'ev NS, Taranenko GS (1986) Raman scattering study of nitric acid solutions. J Appl Spectrosc 44:87-91

[25] Smiechowski M, Stangret J (2008) ATR FT-IR $\mathrm{H}_{2} \mathrm{O}$ spectra of acidic aqueous solutions. Insights about proton hydration. J Mol Strut 878:104-115

[26] Mincher BJ Modolo G, Mezyk SP (2009) Review Article: The Effects of Radiation Chemistry on Solvent extraction 3: A Review of Actinide and Lanthanide Extraction. Solvent Extr Ion Exch 27:579-606 